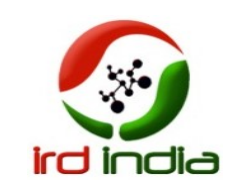

\title{
Classification of MRI Brain Images using K-NN and k-means
}

\author{
V. S. Takate \& P . S. Vikhe \\ Department of Instrumentation \& Control, Pravara Rural Engineering College, Loni Ahmednagar, Maharashtra
}

\begin{abstract}
This paper presents a new approach for diagnosis of brain cancer. Proposed system combines feature extraction techniques with classification and segmentation techniques for diagnosis of the brain as normal or abnormal. The diagnosis based on the classification of magnetic resonance images (MRI).The Method consists of three stages. In the first stage the image is filtered using Discrete Wavelet Transform(DWT) and the features are extracted using Principal component analysis(PCA).In the second stage the segmentation of MRI images are done using K-mean clustering. In the last stage the k-nearest neighbor classifier is used to classify the MRI image as cancerous or non cancerous. Also the twelve properties of extracted features are calculated. Therefore this classification method becomes robust and effective .
\end{abstract}

Keywords - Magnetic resonance images(MRI),Discrete wavelet transform(DWT), Principal component analysis(PCA),K-nearest neighbour (K-NN).

\section{INTRODUCTION}

Brain is a major part of a body. Brain has a very complex structure. That complex structure is protected by a skull. All the functions of body are governed by a Brain. Brain can affected by problem which can cause changes in its normal structure and normal behavior, is called as a Brain cancer. Brain tumor causes abnormal growth of cells in a brain. Brain is a kernel part of the body so diagnosis of brain tumor is very important now a days.

Magnetic resonance imaging is a medical imaging technique. It visualizes internal structure of the brain. MR imaging is a superior to computer tomography for differentiating between tumors and also does not cause harmful ionizing radiation. These MRI images then given as a input to the DWT(Discrete Wavelet Transform). Haar Wavelet is used for filtering images.PCA(Principal component Analysis) is used for reducing features. Then K-NN (K-Nearest Neighbor) Method is used for classification of Brain MRI Images. Then Different twelve properties of Images are calculated such as Accuracy, Sensitivity, Specificity, PPV(Positive predictive value), NPV(Negative predictive value), FDR(False predictive rate), Skewness , Kurtosis, Energy, Entropy, Matthews correlation coefficient, Mean, Variance ,standard deviation etc. In the another part segmentation of MRI Brain images are done using k-means clustering.

\section{METHODOLOGY}

In the proposed system segmentation \& classification of MRI brain images using Fourteen properties are given.

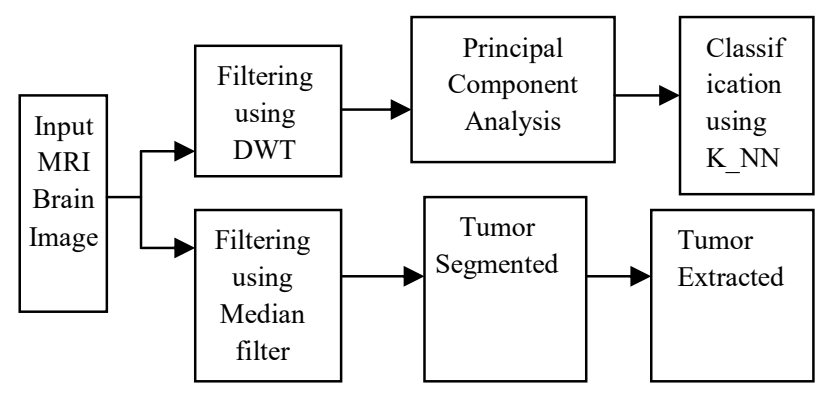

Fig. 2.1: Methodology

The Input MRI brain images are given as a input to the DWT.A two level DWT Harr wavelet is used. Then Principal component analysis is used to reduced the features to a lower level. After PCA the K-NN (Knearest neighbor) method is used to classify the images.

$\mathrm{K}-\mathrm{NN}$ is a non parametric or one of the simple machine learning method of classification. $\mathrm{K}-\mathrm{NN}$ is a one of the simplest classification technique. Classification is done by determining the $\mathrm{k}$ closest training vectors according to a suitable distance metric. Then the input vector which is obtained by PCA is then assigned to a class to which the majority of those $\mathrm{k}$ nearest neighbors belong to. The algorithm for the $\mathrm{k}$ nearest neighbor rule is given below.

Assume that $\mathrm{x}$ will be the unknown feature vector and a distance measure, then

1. Out of $\mathrm{N}$ training vectors, identify the $\mathrm{k}$ nearest neighbors. 
2. Out of these $\mathrm{k}$ samples, identify the number of vectors, ki that belongs to class wi, $\mathrm{i}=1,2$, -M.

3. Then assign $x$ to the class wi with the maximum number $\mathrm{ki}$ of samples.Here we have used the Euclidean distance measure.

We have studied 300 MRI brain images. We have taken MRI database from Harvard Medical School website (http:// med.harvard.edu/AANLIB/, pravara medical college Loni and from www.overcode.yak.net. Following properties are calculated and values of properties of 10 sampled images are plotted here.

1. Kurtosis:- It is a measure of how outlier prone a distribution is.

2. Skewness:- It is a measure of the symmetry of the probability distribution of a real valued random variable.

3. Accuracy:- It is the probability that a diagnostic test is correctly performed.

4. Sensitivity:- It is the probability that a diagnostic test is positive.

5. Specificity:- It is the probability that a diagnostic test is negative.

6. False Discovery rate(FDR):- is a statistical method used in multiple hypothesis testing to correct for multiple comparisons.

7. Positive predictive value(Precision rate):- It is a proportion of positive test results that are true positives.

8. Negative predictive value $(N P V)$ :- is a summary statistic used to describe the performance of a diagnostic testing procedure.

9. Mattews correlation coefficient (MCC):-

The Matthews correlation coefficient is used in machine learing as a measure of the quality of binary (two-class) classifications. It takes into account true and false positives and negatives and is generally regarded as a balanced measure which can be used even if the classes are of very different sizes. The MCC is in essence a correlation coefficient between the observed and predicted binary classifications; it returns a value between -1 and +1 . A coefficient of +1 represents a perfect prediction, 0 no better than random prediction and -1 indicates total disagreement between prediction and observation.

\section{RESULT AND CONCLUSION}

Seven properties are plotted here and Seven are given in tabular form in percentage.

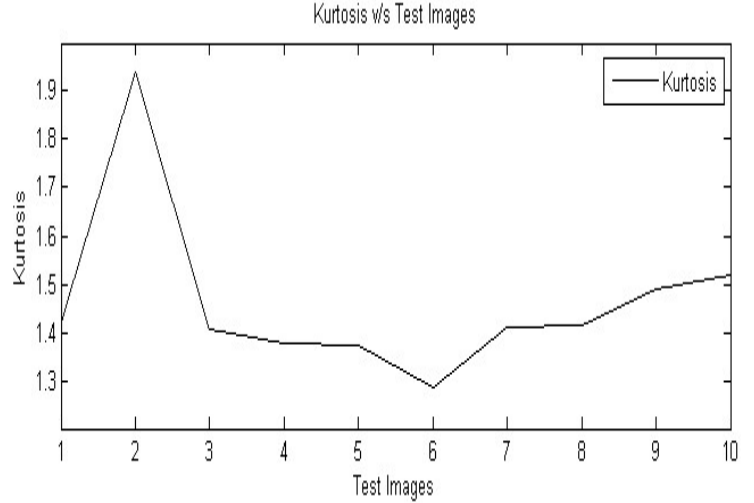

Fig. 2.2: Kurtosis

Skewness w/s Test Images

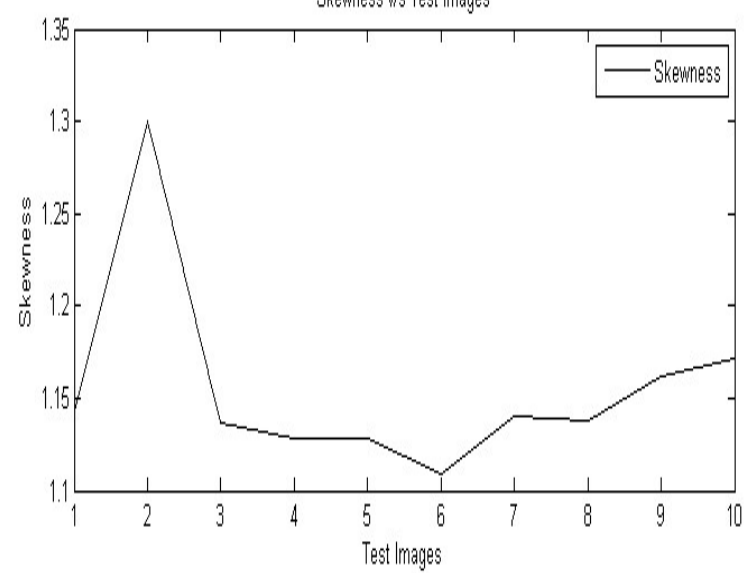

Fig.2.3: Skewness

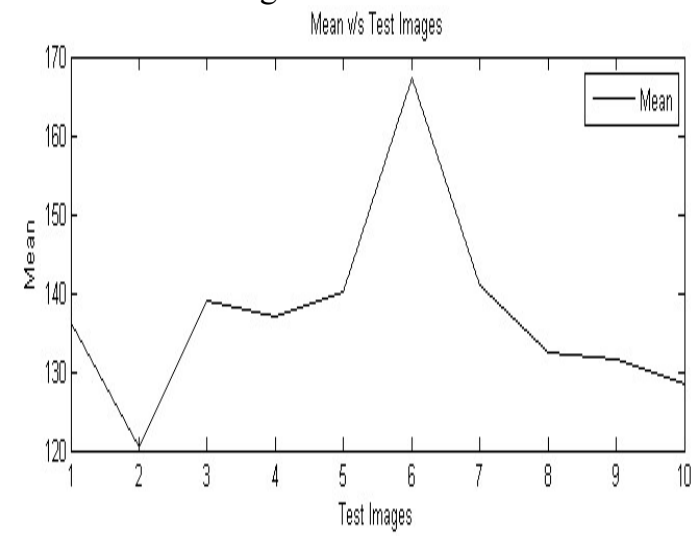

Fig. 2.4: Mean

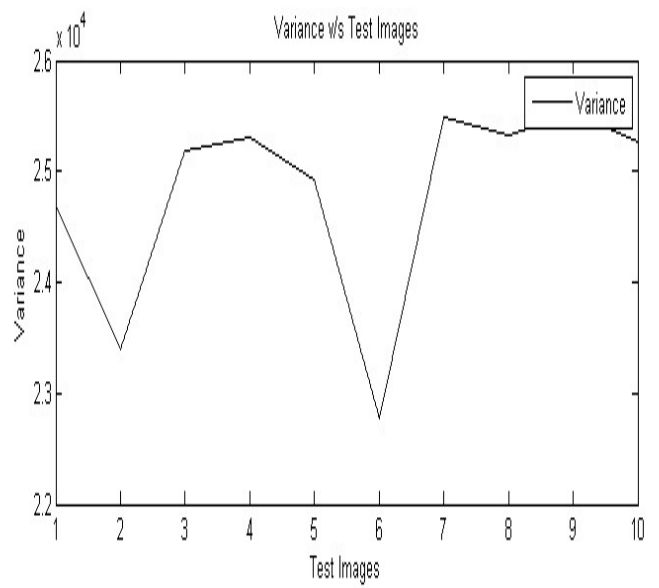

Fig. 2.5: Variance 


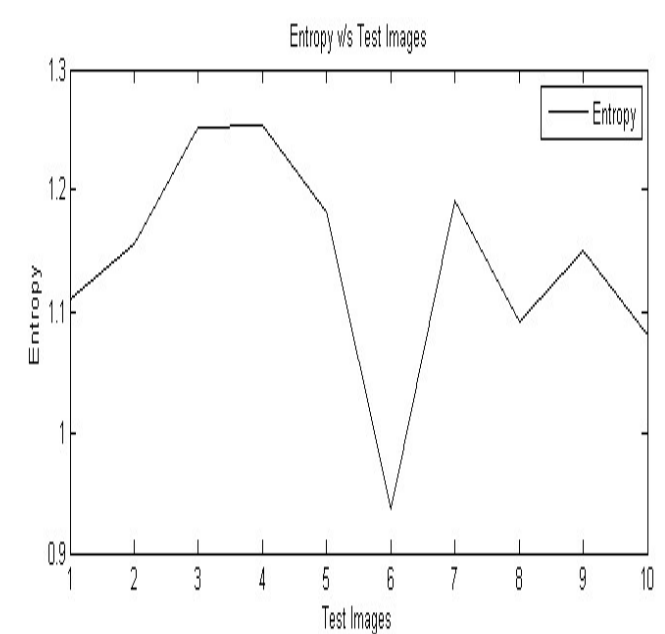

Fig. 2.6: Entropy

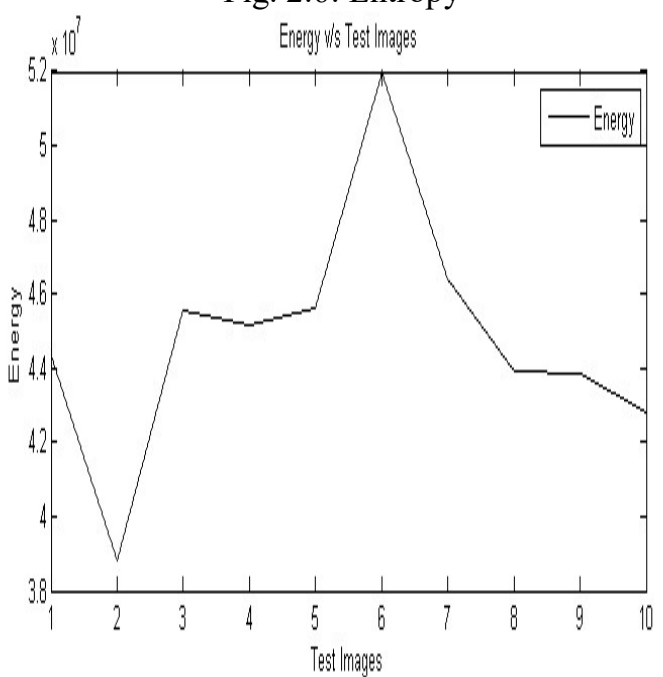

Fig. 2.7: Energy

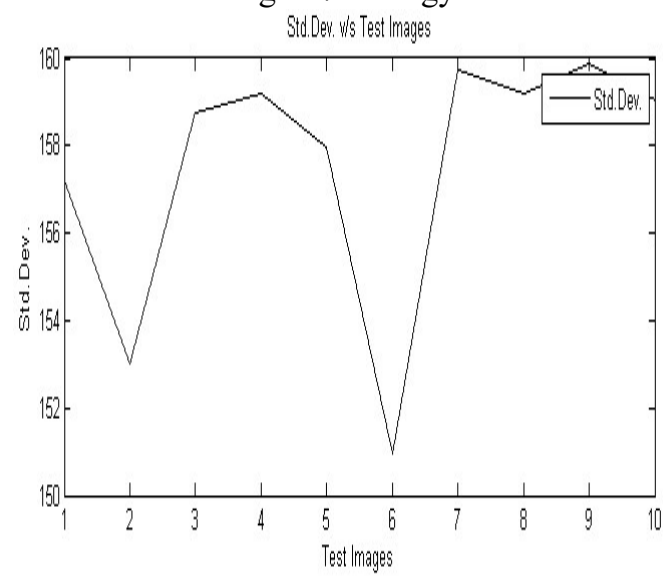

Fig. 2.8: Standard deviation

Following properties are given in tubularform in percentage value.

\begin{tabular}{|c|c|c|c|}
\hline $\begin{array}{c}\text { No. of } \\
\text { samples }\end{array}$ & Accuracy & $\begin{array}{c}\text { Sensiti } \\
\text { vity }\end{array}$ & Specificity \\
\hline 60 & 98.6 & 100 & 90 \\
\hline
\end{tabular}

\begin{tabular}{|c|c|c|c|c|}
\hline $\begin{array}{c}\text { No. of } \\
\text { samples }\end{array}$ & PPV & NPV & FDR & MCC \\
\hline
\end{tabular}

\begin{tabular}{|l|l|l|l|l|}
\hline 60 & 98.4 & 100 & 1.63 & 94.08 \\
\hline
\end{tabular}

In the second part the segmentation of the MRI brain images are done to extract the tumor. Segmentation is a process of partitioning a digital image into multiple segments or set of pixels. Segmentation is used to locate objects and boundaries in the image. So that the tumor extracted and the calculated properties are match with each other. Segmentation is done using kmeans clustering method. Segmentation of Input MRI images are shown here graphically.

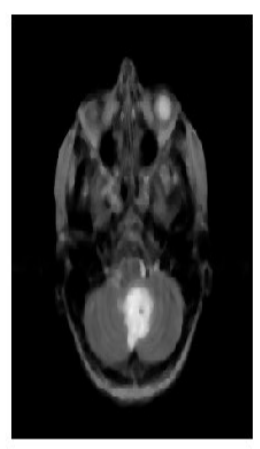

Fig. 2.9: Input MRI Image

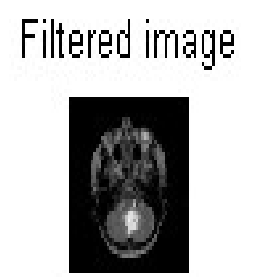

\section{Tumor segmented}

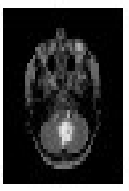

\section{Tumor extracted}

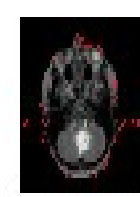

Fig. 2.10: Tumor Segmented

In this way the classification of MRI brain images is done by the above properties whether it is benign or malignant and the tumor region is extracted using MATLAB 7.1 software. 


\section{REFERENCES}

[1] Raghaveer m.rao ajit.s.bopardikar "wavelet transform, introduction to theory and applications" .Pearson education Asia, pp122.,2002.

[2] William K. Pratt, "Digital Image Processing "PRATT Third edition,pp1-15Subra suresh "biomechanics \& biophysics of cancer cells" science direct ,acta material 55,pp 39894014,2007.
[3] Michal w vannier \& John w. Haller "biomedical image segmentation", IEEE journal pp 8186$8821,1 / 98,1998$.

[4] The MATLAB version 7.3 , Toolboxes for Image processing and Wavelet transform.

[5] Rafael Gonzalez, Richard Woods, Steven Eddins "Digital Image Processing using MATLAB 2007". 